\title{
Sniffing Entrapped Humans with Sensor Arrays
}

\author{
Andreas T. Güntner, ${ }^{\dagger}$ Nicolay J. Pineau, ${ }^{\dagger}$ Paweł Mochalski, ${ }^{\ddagger}$ Helmut Wiesenhofer, ${ }^{\ddagger}$ Agapios Agapiou, \\ Christopher A. Mayhew, ${ }^{\ddagger}$ and Sotiris E. Pratsinis ${ }^{*},^{\dagger}$ \\ ${ }^{\dagger}$ Particle Technology Laboratory, ETH Zurich, Zurich CH-8092, Switzerland \\ ${ }^{ \pm}$Institute for Breath Research of the University of Innsbruck, Dornbirn AT-6850, Austria \\ ${ }^{\S}$ Department of Chemistry, University of Cyprus, P.O. Box 20537, Nicosia CY-1678, Cyprus
}

\section{Supporting Information}

ABSTRACT: Earthquakes are lethal natural disasters frequently burying people alive under collapsed buildings. Tracking entrapped humans from their unique volatile chemical signature with hand-held devices would accelerate urban search and rescue (USaR) efforts. Here, a pilot study is presented with compact and orthogonal sensor arrays to detect the breath- and skin-emitted metabolic tracers acetone, ammonia, isoprene, $\mathrm{CO}_{2}$, and relative humidity $(\mathrm{RH})$, all together serving as sign of life. It consists of three nanostructured metal-oxide sensors (Si-doped $\mathrm{WO}_{3}, \mathrm{Si}$ doped $\mathrm{MoO}_{3}$, and Ti-doped $\mathrm{ZnO}$ ), each specifically tailored at the nanoscale for highly sensitive and selective tracer
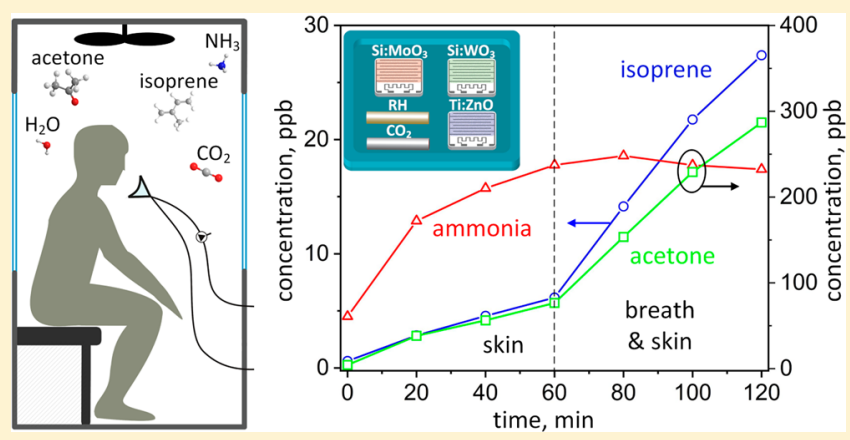
detection along with commercial $\mathrm{CO}_{2}$ and humidity sensors. When tested on humans enclosed in plethysmography chambers to simulate entrapment, this sensor array rapidly detected sub-ppm acetone, ammonia, and isoprene concentrations with high accuracies $(19,21$, and $3 \mathrm{ppb}$, respectively) and precision, unprecedented by portable sensors but required for USaR. These results were in good agreement (Pearson's correlation coefficients $\geq 0.9$ ) with benchtop selective reagent ionization time-of-flight mass spectrometry (SRI-TOF-MS). As a result, an inexpensive sensor array is presented that can be integrated readily into handheld or even drone-carried detectors for first responders to rapidly screen affected terrain.

$\mathrm{R}$ ecent major earthquakes in Mexico (2017), Italy (2017), and Nepal (2015) with thousands of deaths demonstrated once more the severe destructive potential of natural disasters. Earthquakes caused more than 780000 deaths in the past decade, and alarmingly, the number of deaths may increase given progressing urbanization and vulnerability of most populous cities located on fault-lines (e.g., Tokyo, Los Angeles, or Delhi). ${ }^{1}$ Following an earthquake, many victims are entrapped under collapsed buildings and need rapid help, because survival rates drop dramatically within the first hours. ${ }^{2}$ Indispensable for urban search and rescue (USaR) are canines with their superior ability to sniff entrapped humans from their scent. However, their availability and operational time are limited and they are rather stress-sensitive. ${ }^{3}$ Nowadays, specialized equipment (Table S1) is also available to support USaR teams, but these rely mostly on optical and acoustic probes. $^{4}$ As a result, they might not be suitable for rapid sweeping of large areas, especially at limited visible access or with unconscious victims unable to give acoustic signs.

Chemical recognition of the unique volatile signature ${ }^{5,6}$ of humans could improve USaR tools by adding a "third sense", similar to the canines sophisticated nose. Particularly promising to serve as sign of life is the combined detection of breath- and skin-emitted metabolic tracers like acetone, ammonia, and isoprene originating from lipolysis, ${ }^{7}$ protein metabolism, ${ }^{8}$ and cholesterol biosynthesis, ${ }^{9}$ respectively. In fact, recent studies ${ }^{10-13}$ demonstrated that these biomarkers rapidly accumulate near entrapped humans. The employed SRI-TOF-MS ${ }^{11}$ is highly sensitive, selective, fast, and can detect a large range of volatile compounds; however, it lacks portability, is expensive, and requires trained personnel. Therefore, it can hardly be used as portable detector for widespread distribution to first responders. Some other methods can be miniaturized to portable devices, for instance, membrane inlet mass spectrometry (MIMS $)^{12}$ or gas chromatography coupled with ion mobility spectrometry (GC-IMS), ${ }^{10}$ as was reviewed recently. ${ }^{6,14,15}$ Both feature fast response times of few minutes (GC-IMS with rapid multicapillary columns ${ }^{10}$ ) and highly sensitive, selective, and simultaneous detection of various tracers. However, GC-IMS has limited dynamic range ${ }^{13}$ and further miniaturization, power, and cost reductions may be difficult due to indispensable auxiliary systems.

Sensor arrays can be extremely compact, ${ }^{16}$ inexpensive ${ }^{17}$ and are used already as portable devices for indoor air quality, ${ }^{18}$ food spoilage monitoring, ${ }^{19}$ or medical breath analysis. ${ }^{20}$ Even drones or land robots could carry these arrays to rapidly screen

Received: January 16, 2018

Accepted: March 30, 2018

Published: March 30, 2018 


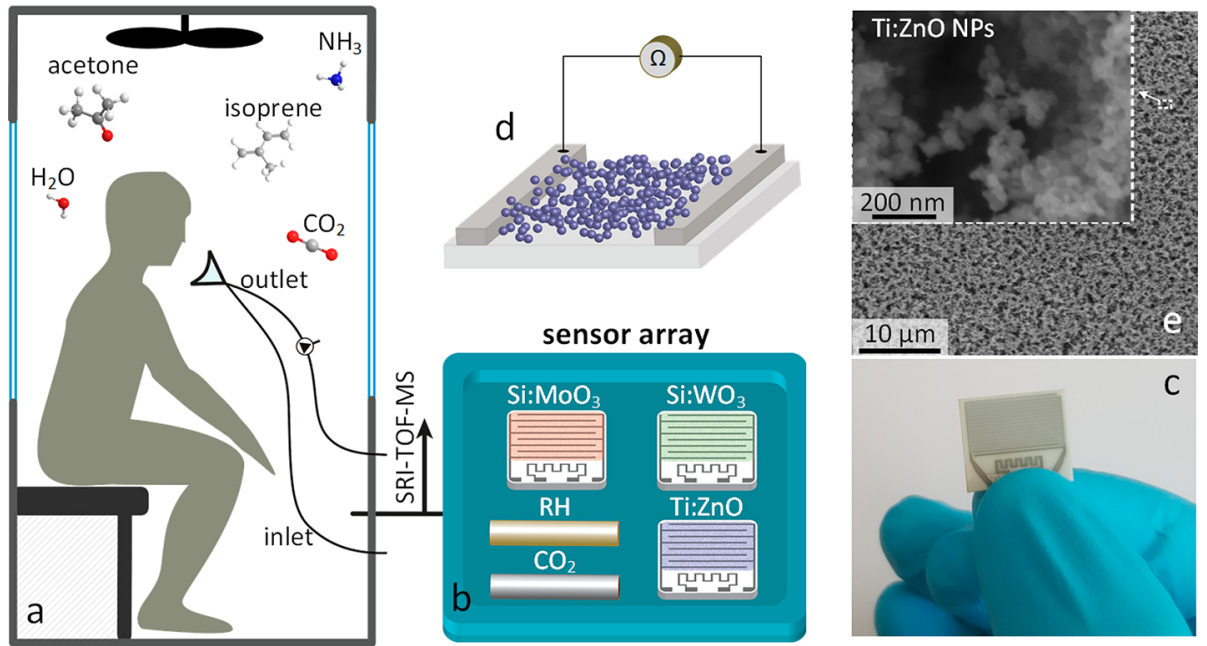

Figure 1. Experimental setup: (a) skin- and breath-borne volatiles of entrapped volunteers accumulate in a plethysmographic chamber. (b) The sensor array consists of three chemoresistive sensors, Si-doped $\mathrm{MoO}_{3}$, Si-doped $\mathrm{WO}_{3}$, and Ti-doped $\mathrm{ZnO}$ to monitor ammonia, acetone, and isoprene, respectively, together with commercial humidity and $\mathrm{CO}_{2}$ sensors. Simultaneous SRI-TOF-MS measurements were used for crossvalidation. (c) Image of a single sensor. (d) The sensing elements consist of highly porous and semiconductive films formed by direct flame deposition of agglomerated/aggregated metal-oxide nanoparticles, as shown by (e) top-view scanning electron microscopy exemplarily for Ti-doped $\mathrm{ZnO}$.

affected areas too dangerous for first responders. Current devices are typically based on a set of highly and broadly sensitive (thus barely selective) chemical sensors mimicking the mammalian olfactory system ${ }^{21}$ (thus frequently called E-noses) to discriminate odors or detect single tracers in simplified laboratory gas mixtures (e.g., formaldehyde ${ }^{22}$ ). However, Enoses lack the accuracy and robustness to sense metabolic tracers at relevant low ppb concentrations ${ }^{5}$ in complex mixtures impeding their application in entrapped human detection ( $>870$ compounds exhaled and $>530$ emitted through skin $^{23}$ ). This is primarily due to the broadly sensitive and rather collinear nature of the applied sensors leading to low discrimination power and susceptibility to environmental confounders, ${ }^{16}$ omnipresent in USaR areas (e.g., from fires, leaked chemicals, etc.).

Here, we present a sensor array based on distinctly selective gas sensors for rapid tracking of entrapped humans (Figure 1a). It consists of three previously developed tailor-made gas sensors, Si-doped $\mathrm{WO}_{3}$, Si-doped $\mathrm{MoO}_{3}$, and Ti-doped $\mathrm{ZnO}$, featuring high sensitivity and selectivity to the metabolic tracers acetone, ${ }^{24}$ ammonia, ${ }^{25}$ and isoprene, ${ }^{26}$ respectively (Figure $1 \mathrm{~b}$ ). These sensors consist of nanostructured, highly porous metaloxide films (Figure 1d,e) that are chemoresistive (i.e., resistance modulated upon interaction with the target analytes) and offer high surface area to detect tracers even at the lowest ppb concentrations. In fact, their lower limits of detection (LOD, at signal-to-noise ratio $=3$ ) are $2.9,50.7$, and $0.7 \mathrm{ppb}$ for acetone, ${ }^{24}$ ammonia, ${ }^{25}$ and isoprene ${ }^{26}$ at $90 \% \mathrm{RH}$, respectively, comparable to GC-IMS ( $30 \mathrm{ppb}$ for acetone ${ }^{10}$ ). Also relatively high analyte concentrations can be detected, e.g., 500 ppm of ammonia with similar $\mathrm{MoO}_{3}$ sensors. ${ }^{27}$ Such sensing films are obtained by direct deposition of flame-made nanoparticles on substrates (Figure 1c) forming finely structured sensing networks, ${ }^{28}$ as shown exemplarily for Ti-doped $\mathrm{ZnO}$ (Figure 1e). Combined with commercial $\mathrm{CO}_{2}$ and $\mathrm{RH}$ sensors, they result in an array with nearly orthogonal sensing characteristics enabling superior discrimination power to accurately detect the chemical signature of humans.
In principle, this array analyses gas mixtures (here, human volatile emissions) with each sensor individually and response times $<3 \mathrm{~min}$ (Figure S1, inset). The generated signals are processed with a statistical model ${ }^{22}$ to estimate tracer concentrations by combinatorial selectivity ${ }^{21}$ with enhanced accuracy compared to single sensors (Figure 2). For acetone,

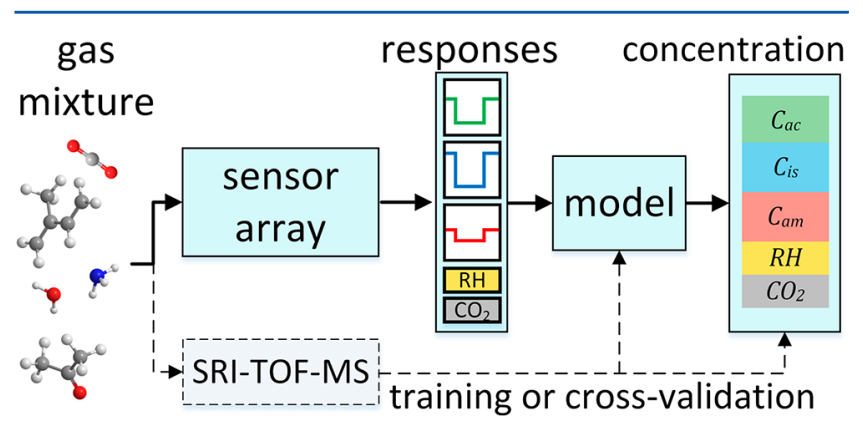

Figure 2. Sensor array concept: Gas mixtures containing the breath and skin emitted tracers are analyzed by each sensor individually and their signals are converted by a statistical model ${ }^{22}$ to analyte concentrations. This model is initially "trained" with data of four volunteers and tested on five volunteers.

ammonia, and isoprene, a multivariate linear regression (MVLR) model $^{29}$ is applied (please see Methods in the Supporting Information) matching the linear response characteristics of these sensors at sub-ppm analyte concentrations. $^{24-26}$ Note that these become nonlinear at higher concentrations which can be addressed in the model. To determine model coefficients, the measured data were separated into a "training" and an independent validation set.

Next, we applied the sensor array to monitor volatile compounds related to human chemical signatures (or body odor). Therefore, nine volunteers (Table S2 for physiological data) were enclosed individually in a plethysmographic chamber to mimic entrapment conditions. ${ }^{11}$ The testing course for each volunteer lasted $120 \mathrm{~min}$, first with only skin (0-60 $\mathrm{min}$ ) followed by breath and skin (60-120 min) emissions into 

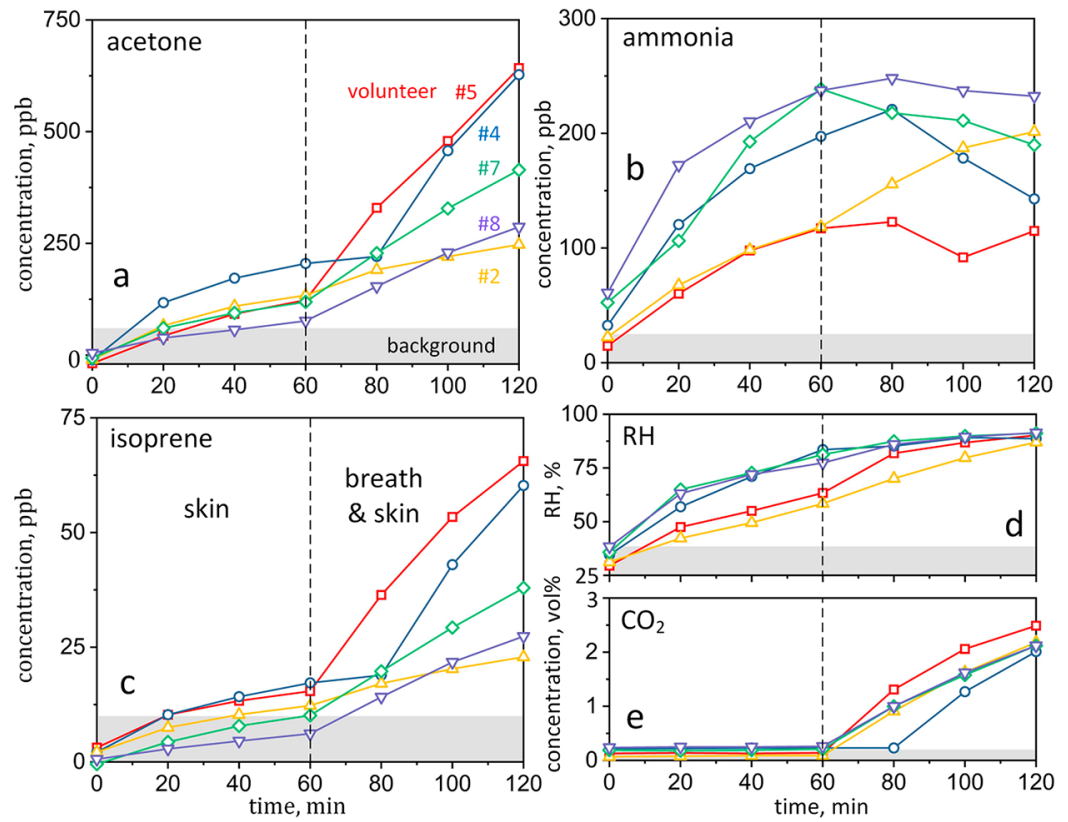

Figure 3. Sensor array measurements of acetone (a), ammonia (b), isoprene (c), $\mathrm{RH}(\mathrm{d})$, and $\mathrm{CO}_{2}$ (e) concentrations of five volunteers as a function of entrapment time. Skin only (0-60 min) followed by skin and breath (60-120 min) emissions were studied separately. In the case of volunteer no. 4 (circles), skin (only) emissions lasted accidentally for $80 \mathrm{~min}$. Room air (background) concentrations are indicated in gray.
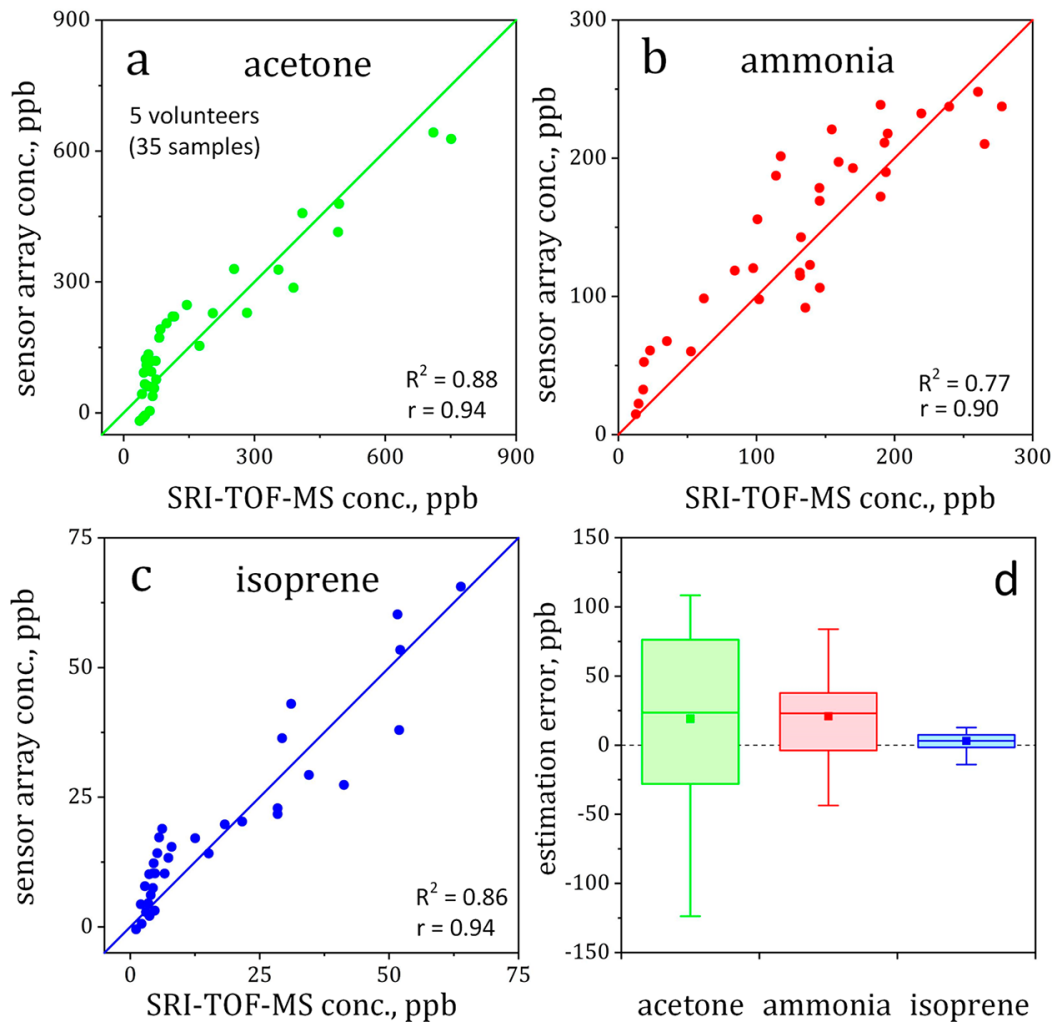

Figure 4. Scatter plots indicating correlations between sensor array and SRI-TOF-MS for acetone (a), ammonia (b), and isoprene (c) along with their corresponding Pearson's correlation coefficients $(r)$ and coefficients of determination $\left(R^{2}\right)$. (d) Box-and-whisker plot of sensor array estimation errors. Medians and means are shown as lines and squares, respectively. The boxes represent the first and third quartiles and whiskers indicate the full ranges.

the chamber. These emissions are investigated separately to better understand the release pathways of target tracers. Figure 3 shows the corresponding sensor array estimated acetone (a), ammonia (b), isoprene (c), $\mathrm{RH}$ (d), and $\mathrm{CO}_{2}$ (e) concentration profiles of five volunteers (with individual colors and symbols) when measured every $20 \mathrm{~min}$. Note that the data of the other four randomly selected volunteers were used for "training" of the MVLR model to achieve minimal estimation errors at the smallest sample size (please see Figure S2 for errors at other sample sizes and Figure $\mathrm{S} 3 \mathrm{a}-\mathrm{c}$ for concentration 
profiles of all volunteers). The room air (background) concentration range for each tracer are indicated in gray in Figure 3.

In a typical case (e.g., volunteer no. 7, diamonds in Figure 3), acetone, isoprene, and $\mathrm{CO}_{2}$ change only little during skin emission $(0-60 \mathrm{~min})$ while they increase significantly when also exhaled (60-120 min), as detected by the sensor array and consistent with SRI-TOF-MS (Figure S3d,f). As a result, these tracers can indicate human presence (breath and skin emissions) rather early as their concentrations rapidly exceed background levels. Furthermore, the concentration slopes (and thus emission rates) among the volunteers vary significantly, especially for acetone and isoprene when emitted from breath and skin simultaneously (Figure 3a,c, $t>60 \mathrm{~min}$ ). This is expected due to biological variations of breath acetone ${ }^{30}$ and isoprene $^{31}$ that are caused, for instance, by different metabolic states. For volunteer no. 5 (Figure 3a, squares), higher acetone emissions should indicate intensified ketogenesis, ${ }^{7}$ reasonable after $8 \mathrm{~h}$ of fasting prior to the experiment and a likely case for victims after prolonged entrapment. In fact, previous breath studies on fasting subjects revealed increasing acetone levels during exercise and rest indicating enhanced fat oxidation, as confirmed by a blood assay. ${ }^{20}$ Despite these individual differences, acetone, isoprene and $\mathrm{CO}_{2}$ concentrations are distinguished clearly from the background (Figure 3, grayshaded) for all volunteers after $120 \mathrm{~min}$ of entrapment, so human presence in the plethysmographic chamber is recognized unambiguously.

Finally, it is worth discussing volunteer no. 4 (Figure 3, circles) who removed by mistake the mask outlet after $80 \mathrm{~min}$ (instead of $60 \mathrm{~min}$ ), so his phase of only skin emissions lasted longer. The sensor array "recognized" the prolonged skin emissions correctly. In fact, the increase in acetone and isoprene concentrations was delayed (Figure 3a,c), in excellent agreement with the SRI-TOF-MS (Figure S3d,f). This shows nicely how the sensor array can pick up individual tracer concentration profiles, even when deviating from the measurement protocol.

On the other hand, ammonia and $\mathrm{RH}$ increase significantly during skin emission and differ from the background even after short entrapment. Later, both tend to level off and ammonia may even decrease in some cases (e.g., volunteer no. 4 and 7), as confirmed by SRI-TOF-MS (Figure S3e). This may be related to absorption of hydrophilic ammonia in water films. In fact, water condensation on the colder chamber walls was apparent at high $\mathrm{RH}(>80 \%)$. Only for volunteer no. 2 (Figure $3 \mathrm{~b}$, triangles), ammonia levels increase steadily, consistent with slower rising $\mathrm{RH}$ levels (Figure $3 \mathrm{~d}$ ) that might be associated with his skinny physique (lowest weight at normal height, Table S2). Note that tracer concentrations may be altered also by construction materials, such as alumina that retains hydrophilic molecules, ${ }^{32}$ or other background effects (e.g., fire or garbage) possibly present in USaR environments. Nevertheless, ammonia is still a promising human tracer due to its high skin emission rate and thus rapid accumulation in the vicinity of a person.

To cross-validate the sensor array's accuracy, all measured concentrations are compared to SRI-TOF-MS. Figure 4 shows the scatter plots of acetone (a), ammonia (b), and isoprene (c) as measured by the sensor array and SRI-TOF-MS for the five volunteers (35 samples). Both methods correlate strongly for all analytes (Pearson's correlation coefficients $\geq 0.9, p<0.05$, Table S2 for each volunteer) with outstanding accuracy and precision (Figure 4d). Specifically, the accuracies are 19, 21, and $3 \mathrm{ppb}$ for breath- and skin-emitted acetone, ammonia, and isoprene, respectively (Figure $4 \mathrm{~d}$, filled squares). This is remarkable considering on one hand the sensor array's simple, inexpensive, and compact design compared to SRI-TOF-MS and on the other hand, the complexity of the analyzed gas mixture with strong variation of composition and conditions (e.g., $\mathrm{RH}$ from 28 to $90 \%$ or temperature from 19.5 to $27^{\circ} \mathrm{C}$, Figure S4). Also, it is significantly better (mean errors 4-28 times lower) than single sensors (compare Figure S5b to Figure $4 \mathrm{~d}$ and note different ordinate scale) likely due to the higher discrimination power through the nearly orthogonal array design. This highlights the potential of sensor arrays to detect breath- and skin-emitted tracer signatures, relevant for entrapped human detection.

Note that there is a mismatch between sensor array and SRITOF-MS for acetone (Figure 4a) below $150 \mathrm{ppb}$ and isoprene (Figure 4c) below $10 \mathrm{ppb}$. These deviations occur during the skin-emission phase $(0-60 \mathrm{~min})$ as the sensor array estimates higher acetone (Figure 3a) and isoprene (Figure 3c) concentrations than SRI-TOF-MS (Figure S 3d,f). Errors may be caused by sensor cross-sensitivities to other compounds, for instance, the more than 530 skin-emitted volatiles ${ }^{23}$ where less acetone and isoprene are released. Furthermore, disaster environments may contain high concentrations of hydrogen and $\mathrm{CO}$ and even humans exhale them at concentrations of several ppm. ${ }^{33,34}$ However, their interference seems not that significant, as indicated by the strong correlations between sensor array and SRI-TOF-MS for the target tracers (Figure $4 \mathrm{a}-\mathrm{c}$ ) and individual volunteers (Table S2). This is consistent with single $\mathrm{Si}$-doped $\mathrm{MoO}_{3}{ }^{25}$ sensors that had shown no response to $\mathrm{CO}$.

Reliable detection of entrapped humans requires multitracer detection since single compounds are affected too easily by other sources (e.g., ammonia by $\mathrm{RH}$ in Figure $3 \mathrm{~b}$ or $\mathrm{CO}_{2}$ by fire). Consequently, all tracers need to be evaluated simultaneously for recognition of patterns indicative of human presence. Therefore, the results were visualized by normalizing the analyte concentration in the chamber $\left(c_{c}\right)$ to its background $\left(c_{\mathrm{b}}\right)$ and associating a color code to their ratios $\left(c_{\mathrm{c}}\right)$ $\left.c_{b}\right)$. The color map in Figure 5 shows the evolution of the five volunteers (average $c_{\mathrm{c}} / c_{\mathrm{b}}$ ) during entrapment. Most impor-

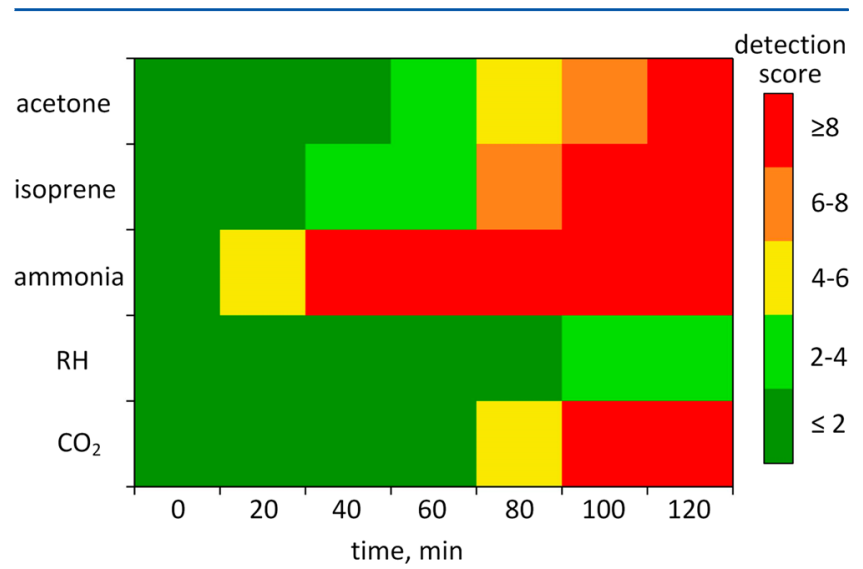

Figure 5. Color map indicating human detection by their skin $(0-60$ $\mathrm{min})$ followed by breath and skin (60-120 $\mathrm{min}$ ) emissions. For each analyte, a detection score $\left(c_{c} / c_{b}\right)$ is calculated representing the ratio of average concentration in the background $\left(c_{\mathrm{b}}\right)$ and chamber air $\left(c_{\mathrm{c}}\right)$ in the presence of five volunteers. 
tantly, all tracers (except for $\mathrm{RH}$ ) stand out from the background $\left(c_{\mathrm{c}} / c_{\mathrm{b}}>4\right)$ and develop a distinct pattern after 120 min corresponding to a short entrapment in a typical USaR mission. This pattern seems quite characteristic for humans as can be seen from the rather similar color maps of individual volunteers in Figure S6. As a result, the on-site detection of target tracers could act as an early indication of human presence under the ruins of collapsed buildings though further validation under field conditions is needed. Note that other tracers could be included to enhance robustness by introducing additional distinctly selective sensors into the modular array design.

In summary, a novel sensor array has been developed for rapid detection of entrapped humans from their volatile compound emissions. By choosing tailor-made and nanostructured, chemoresistive gas sensors with distinct selectivities, this array featured nearly orthogonal sensing characteristics resulting in unprecedented sensitivity, discrimination power, and robustness against other breath- and skin-emitted compounds. This facilitated the accurate detection of breathand skin-emitted acetone, ammonia, and isoprene concentrations even at the lowest ppb levels, as confirmed by a benchtop SRI-TOF-MS. This is superior to conventional sensor arrays that detect only response patterns without identifying analytes. When finally applied on entrapped volunteers, the detector recognized human presence and even distinct behavior (volunteer no. 4) by multitracer assessment. This pilot study indicates that such sensor arrays could be quite effective during real USaR and should be tested in collaboration with first responders. Therein, positioning of (single and multiple) entrapped humans, effects of physiological, pathological, and other conditions (e.g., injuries, dehydration, asphyxiation, shock, cosmetics) and false positive alarms from external confounders (e.g., leaked chemicals or fire) need to be considered. Finally, this sensor array featured a compact size to be incorporated easily into hand-held or even drone-carried detectors.

\section{ASSOCIATED CONTENT}

\section{S Supporting Information}

The Supporting Information is available free of charge on the ACS Publications website at DOI: 10.1021/acs.analchem.8b00237.

Experimental methods, exemplary sensor responses, average sensor array estimation errors at different sample sizes, sensor array and SRI-TOF-MS tracer estimations for 9 volunteers, $\mathrm{RH}$ and temperature profiles, color map for each volunteer, applied human tracking technologies in USaR, and physiological data of the volunteers and their individual correlation coefficients between sensor array and SRI-TOF-MS for breath- and skin-emitted tracers are provided (PDF).

\section{AUTHOR INFORMATION}

\section{Corresponding Author}

*E-mail: pratsinis@ptl.mavt.ethz.ch.

\section{ORCID $\odot$}

Andreas T. Güntner: 0000-0002-4127-752X

\section{Notes}

The authors declare no competing financial interest.

\section{ACKNOWLEDGMENTS}

This study was financially supported by the Swiss National Science Foundation (Grant No. 200021_159763/1 and 206021 170729) and the European Union's Horizon 2020 research and innovation program (Grant No. 644031). P.M. gratefully acknowledges additional funding by the Austrian Research Promotion Agency (FFG) for the program KIRAS Security Research under Grant DHS-AS 859586 and the Austria Science Fund (FWF, Grant No. P24736-B23). This work is part of the Zurich Exhalomics project under the umbrella of University Medicine Zurich/Hochschulmedizin Zürich. The authors thank S. Abegg (ETH Zurich) for stimulating discussions. This research received the Best Poster Award during the 2017 European Aerosol Conference Aug. 27-Sept. 1 in Zurich, Switzerland.

\section{REFERENCES}

(1) Bartels, S. A.; VanRooyen, M. J. Lancet 2012, 379 (9817), 748757.

(2) Schultz, C. H.; Koenig, K. L.; Noji, E. K. N. Engl. J. Med. 1996, 334 (7), 438-444.

(3) Wong, J.; Robinson, C. Urban Search and Rescue Technology Needs: Identification of Needs. Federal Emergency Management Agency (FEMA) and the National Institute of Justice (NIJ), 2004.

(4) PROEYE System 951-S model 2010, http://www.ads.com.tr/ safety/en/sar/proeye/sys951.htm, accessed November 2017.

(5) Agapiou, A.; Amann, A.; Mochalski, P.; Statheropoulos, M.; Thomas, C. L. P. TrAC, Trends Anal. Chem. 2015, 66, 158-175.

(6) Giannoukos, S.; Brkić, B.; Taylor, S.; Marshall, A.; Verbeck, G. F. Chem. Rev. 2016, 116 (14), 8146-8172.

(7) Kalapos, M. P. Biochim. Biophys. Acta, Gen. Subj. 2003, 1621 (2), 122-139.

(8) Risby, T. H.; Solga, S. F. Appl. Phys. B: Lasers Opt. 2006, 85 (23), 421-426.

(9) Deneris, E. S.; Stein, R. A.; Mead, J. F. Biochem. Biophys. Res. Commun. 1984, 123 (2), 691-696.

(10) Vautz, W.; Slodzynski, R.; Hariharan, C.; Seifert, L.; Nolte, J.; Fobbe, R; Sielemann, S.; Lao, B. C. Anal. Chem. 2013, 85 (4), 21352142.

(11) Mochalski, P.; Unterkofler, K.; Hinterhuber, H.; Amann, A. Anal. Chem. 2014, 86 (8), 3915-3923.

(12) Giannoukos, S.; Brkić, B.; Taylor, S.; France, N. Anal. Chem. 2014, 86 (2), 1106-1114.

(13) Mochalski, P.; Wiesenhofer, H.; Allers, M.; Zimmermann, S.; Güntner, A. T.; Pineau, N. J.; Lederer, W.; Agapiou, A. J. Chromatogr. B: Anal. Technol. Biomed. Life Sci. 2018, 1076, 29-34.

(14) Snyder, D. T.; Pulliam, C. J.; Ouyang, Z.; Cooks, R. G. Anal. Chem. 2016, 88 (1), 2-29.

(15) Cumeras, R.; Figueras, E.; Davis, C.; Baumbach, J. I.; Gracia, I. Analyst 2015, 140 (5), 1376-1390.

(16) Röck, F.; Barsan, N.; Weimar, U. Chem. Rev. 2008, 108 (2), $705-725$.

(17) Askim, J. R.; Mahmoudi, M.; Suslick, K. S. Chem. Soc. Rev. 2013, 42 (22), 8649-8682.

(18) Sensirion Multi-Pixel Gassensor SGP30, https://www.sensirion. com/de/umweltsensoren/gassensoren/, accessed January 2018.

(19) Li, Z.; Suslick, K. S. ACS Sens. 2016, 1 (11), 1330-1335.

(20) Güntner, A. T.; Sievi, N. A.; Theodore, S. J.; Gulich, T.; Kohler, M.; Pratsinis, S. E. Anal. Chem. 2017, 89 (19), 10578-10584.

(21) Persaud, K.; Dodd, G. Nature 1982, 299 (5881), 352-355.

(22) Güntner, A. T.; Koren, V.; Chikkadi, K.; Righettoni, M.; Pratsinis, S. E. ACS Sens. 2016, 1 (5), 528-535.

(23) De Lacy Costello, B.; Amann, A.; Al-Kateb, H.; Flynn, C.; Filipiak, W.; Khalid, T.; Osborne, D.; Ratcliffe, N. M. J. Breath Res. 2014, 8 (1), 014001.

(24) Righettoni, M.; Tricoli, A.; Pratsinis, S. E. Anal. Chem. 2010, 82 (9), 3581-3587. 
(25) Güntner, A. T.; Righettoni, M.; Pratsinis, S. E. Sens. Actuators, B 2016, 223, 266-273.

(26) Güntner, A. T.; Pineau, N. J.; Chie, D.; Krumeich, F.; Pratsinis, S. E. J. Mater. Chem. B 2016, 4 (32), 5358-5366.

(27) Mutschall, D.; Holzner, K.; Obermeier, E. Sens. Actuators, B 1996, 36 (1-3), 320-324.

(28) Mädler, L.; Roessler, A.; Pratsinis, S. E.; Sahm, T.; Gurlo, A.; Barsan, N.; Weimar, U. Sens. Actuators, B 2006, 114 (1), 283-295.

(29) Mardia, K. V.; Kent, J. T.; Bibby, J. M. Multivariate Analysis; Academic Press: London, 1979.

(30) Spanel, P.; Dryahina, K.; Rejskova, A.; Chippendale, T. W. E.; Smith, D. Physiol. Meas. 2011, 32 (8), N23-N31.

(31) King, J.; Kupferthaler, A.; Unterkofler, K.; Koc, H.; Teschl, S.; Teschl, G.; Miekisch, W.; Schubert, J. J. Breath Res. 2009, 3 (2), 027006.

(32) van den Broek, J.; Güntner, A. T.; Pratsinis, S. E. ACS Sens. 2018, 3 (3), 677-683.

(33) Deveci, S. E.; Deveci, F.; Açik, Y.; Ozan, A. T. Respir. Med. 2004, 98 (6), 551-556.

(34) Perman, J. A.; Modler, S.; Barr, R. G.; Rosenthal, P. Gastroenterology 1984, 87 (6), 1358-1363. 